\title{
HPV strain distribution in patients with genital warts in a female population sample
}

\author{
DANIEL BODA ${ }^{1,2}$, MONICA NEAGU $^{3,4}$, CAROLINA CONSTANTIN $^{3}$, RAZVAN NICOLAE VOINESCU $^{5}$, \\ CONSTANTIN CARUNTU ${ }^{1}$, SABINA ZURAC ${ }^{6}$, DEMETRIOS A. SPANDIDOS ${ }^{7}$, \\ NIKOLAOS DRAKOULIS ${ }^{8}$, DIMITRIOS TSOUKALAS ${ }^{9}$ and ARISTIDES M. TSATSAKIS ${ }^{9}$

\begin{abstract}
${ }^{1}$ Dermatology Research Laboratory, 'Carol Davila' University of Medicine and Pharmacy, Bucharest 050474; ${ }^{2}$ Department of Dermatology, Carol Medical Center, Bucharest 020915; 3 Department of Immunology, 'Victor Babes' National Institute of Pathology, Bucharest 050096; ${ }^{4}$ Faculty of Biology, University of Bucharest, Bucharest 050095; ${ }^{5}$ The Institute for World Economy, Bucharest 050711; ${ }^{6}$ Department of Pathology, 'Carol Davila' University of Medicine and Pharmacy, Colentina University Hospital, Bucharest 020125, Romania; ${ }^{7}$ Laboratory of Clinical Virology,

Medical School, University of Crete, Heraklion 71409; ${ }^{8}$ Research Laboratory of Clinical Pharmacology and Pharmacogenomics, Faculty of Pharmacy, National and Kapodistrian University of Athens, Athens 157 71;

${ }^{9}$ Department of Forensic Sciences and Toxicology, Medical School, University of Crete, Heraklion 71003, Greece
\end{abstract}

Received May 18, 2016; Accepted July 15, 2016

DOI: $10.3892 / 01.2016 .4903$

\begin{abstract}
The incidence of human papillomavirus (HPV) in the human cancer domain is still a subject of intensive study. In this study, we examined cervical swab samples from 713 females with genital warts, and tested the samples for high- and low-risk genital HPV. HPV genotyping was assessed using a Genotyping test that detects HPV by the amplification of target DNA using polymerase chain reaction and nucleic acid hybridization. In total, we detected 37 anogenital HPV DNA genotypes [6, 11, $16,18,26,31,33,35,39,40,42,45,51,52,53,54,55,56,58,59$, 61, 62, 64, 66, 67, 68, 69, 70, 71, 72, 73 (MM9), 81, 82 (MM4), 83 (MM7), 84 (MM8), IS39 and CP6108] and investigated the incidence of these genotypes in the patients with genital warts. We found differences in the distribution of high-/low-risk strains and the incidence of high-risk strains was found to occur mainly in females under 35 years of age. The data from our study suggest that a detailed oral, rectal and genital identification of high-risk strains should be performed to visualize the entire pattern of possible triggers of carcinogenesis.
\end{abstract}

\section{Introduction}

The burden of cervical cancer, which is the main type of cancer related to human papillomavirus (HPV) infection, is substantially higher in Central and Eastern Europe compared

Correspondence to: Professor Monica Neagu, Department of Immunology, 'Victor Babes' National Institute of Pathology, 99-101 Splaiul Independentei, Bucharest 050096, Romania

E-mail: neagu.monica@gmail.com

Key words: carcinogenesis, cervical cancer, genital warts, HPV genotyping with the rest of Europe, with increasing trends of incidence and mortality in several countries. However, several gaps in knowledge exist concerning the incidence and associated mortality of other HPV-related cancers. The same is true for HPV prevalence and type distribution among women with HPV-related cancers other than cervical cancer and in the general female population (1).

Limited data are available regarding HPV incidence in Romania and in terms of mortality due to cervical cancer, Romania ranks first in Europe, recording 6.3-fold more deaths from cervical cancer than the mean registered number in other European countries. Although vaccination campaigns were launched by health officials in Romania, the acceptance rate remained low and thus the programs were discontinued. A successful vaccination program requires a high rate of acceptance and accurate information for health professionals and parents (2). In 2014, Salavastru et al published an epidemiological study stating that genital infection with HPV has become one of the most frequently viral sexually transmitted diseases. The infection may remain asymptomatic, may take the form of external genital warts or may give raise to cervical cancers. The aim of that study was to assess the frequency of patients with genital warts, obtaining data from five tertiary referral dermatological units in Romania, and to compare the results with outpatient data reported by all Romanian hospitals (3). The study reported data obtained for the year 2012, with 952 patients (731 females and 221 males) with 26 males under 20 years of age and 251 female patients in the age group of 0-20 years. In the overall population (males and females combined) the total number of genital warts cases registered at the hospital emergency rooms in the five centers, was approximately $30 \%$. In that epidemiological study though, no data were obtained regarding HPV cervical or urethral genotyping, the incidence of high-risk HPV strains versus low-risk strains and the possible effects on cervical cancer (3). 
In this study, in order to establish the actual high-risk strain distribution in a female population, we assessed the incidence of HPV strains through HPV genotyping in patients with genital warts to highlight the particular pattern of this tumorigenesis related-virus in order to obtain a better understanding of the possible effects in time upon carcinogenesis.

\section{Materials and methods}

A total of 713 female patients with genital warts, treated at Medicover Healthcare Centre (Bucharest, Romania), from January to December, 2015 comprised the patient group and cervical swabs were obtained from these patients. All patients provided written informed consent prior to obtaining the samples, and this study was approved by the Ethics Committee of Carol Medical Center, Bucharest, Romania. Cervical cells were collected in PreservCyt ${ }^{\circledR}$ Solution (Cytyc Corp., Boxborough, MA, USA) and then analyzed. HPV genotyping was assessed using LINEAR ARRAY ${ }^{\circledR}$ HPV Genotyping test (Roche Diagnostics, Indianapolis, IN, USA). Briefly, the test detects HPV by the amplification of target DNA using polymerase chain reaction (PCR) and nucleic acid hybridization as follows:

The standard kit was developed according to previously published protocols (4). Briefly, the samples were prepared for PCR by standard protocols by digestion with proteinase $\mathrm{K}$ and $1 \%$ Laureth-12, spun and heated to $95^{\circ} \mathrm{C}$ for $10 \mathrm{~min}$ residual protease denaturation. The samples were centrifuged and $5 \mu \mathrm{l}$ was used for each PCR assay. PCR amplification was performed according to the manufacturer's recommendations. Briefly, a working master mix was prepared and added into each reaction tube. Subsequently, $50 \mu \mathrm{l}$ of each processed sample was added to the appropriate amplification tubes containing the working master mix. The PCR cycling conditions were as indicated by the manufacturer. Quality control consisted of $2 \mu \mathrm{l}$ of the amplicon electrophoresed on a 1.5\% Flash gel (Lonza Inc., Rockland, ME, USA) and if correct banding was present, genotyping followed. HPV genotyping was performed according to the manufacturer's recommendations. A total of $75 \mu 1$ of denatured amplicon was added to each standard labeled strip, and several incubation and washing cycles were performed at $53^{\circ} \mathrm{C}$ for $30 \mathrm{~min}$. Subsequently, $4 \mathrm{ml}$ of conjugate were added to each well containing a strip, followed by incubation for $30 \mathrm{~min}$. After washing, $4 \mathrm{ml}$ of working substrate were added to each well, followed by incubation. After the strips were dried, the HPV genotyping calls were made using the detectable hybridization bands provided by the manufacturer. The test detects 37 anogenital HPV DNA genotypes [6, 11, 16, 18, 26, 31, 33, 35, $39,40,42,45,51,52,53,54,55,56,58,59,61,62,64,66,67,68$, 69, 70, 71, 72, 73 (MM9), 81, 82 (MM4), 83 (MM7), 84 (MM8), IS39 and CP6108].

Statistical analysis was performed using ANOVA and a value of $\mathrm{P}<0.05$ was considered to indicate a statistically significant difference. The results are presented as a percentage of the distribution and age incidence in various groups using the Prism and Excel programs.

\section{Results}

According to the literature (1-3), there is a panel of HPV high-risk strains, namely 16, 18, 31, 33, 35, 39, 45, 51, 52, 56, 58,

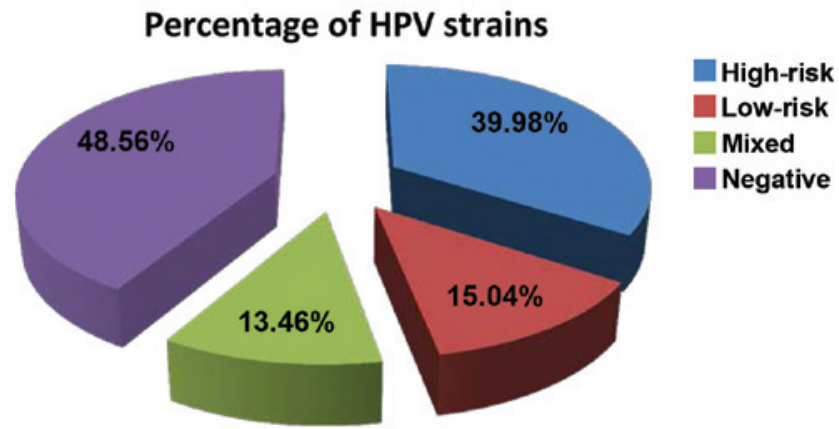

Figure 1. Human papillomavirus (HPV) strain distribution in the investigated study population: percentage of strain type reported in all cases investigated.

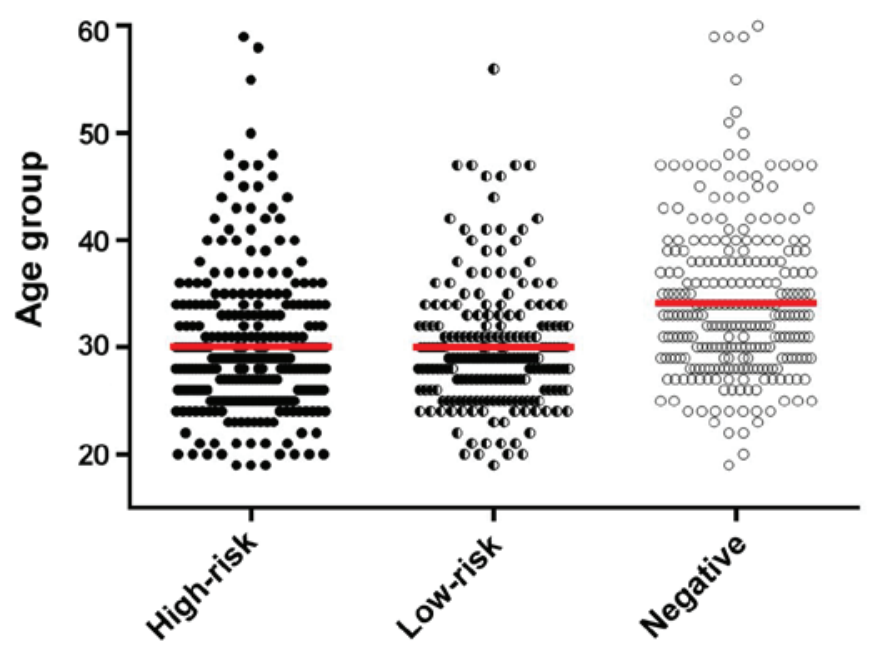

Figure 2. Age distribution in the investigated group in terms of high/low and negative strain identification.

59 and 68, while the panel comprising 26, 40, 42, 53, 54, 55, 61, $62,64,66$ and 67 is considered low-risk. In the patients enrolled in our study who were diagnosed with genital warts, we found that although there was a clear-cut difference between the groups with the high- versus the low-risk strains, there are also a group of patients (not quite negligible) that had both strain types.

When assessing the samples of the patient group (Fig. 1), approximately half of the females examined tested as negative for any HPV strains, while the low-risk strains only were found in approximately $15 \%$ of all cases. Almost $85 \%$ of the study population tested positive either for the high-risk strains or for mixed strains (high + low-risk strains). This last subpopulation of tested females comprised the group that had exhibited a risk pattern, and in terms of numbers, this covered almost half of the population, this latter subpopulation being the main group with a significant risk of developing cancerous lesions.

When assessing the age distribution of all the tested female patients (Fig. 2), we found that as regards the mean age of the low- and high-risk female patient groups, there was no statistically significant difference, while in the group that tested negative there is a clear-cut increase as in mean age. When examining the statistical significance as regards the predisposition for cancer associated with high-risk HPV in terms of female age (Fig. 3) we observed that there was a clear tendency 


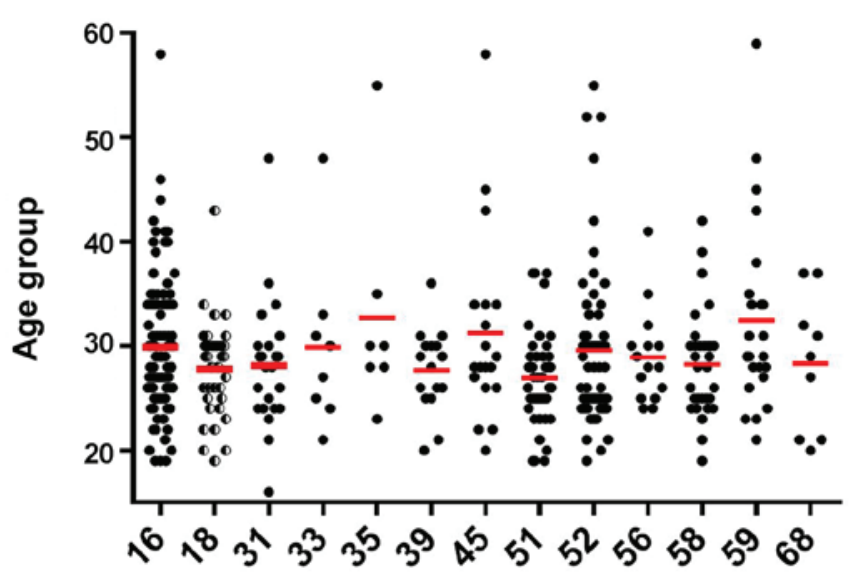

High-risk strains

Figure 3. Age distribution among human papillomavirus high-risk strains.

towards the high-risk HPV strains in patients $<35$ years of age, with no actual preponderance among the different high-risk strains.

\section{Discussion}

Over the past years, the healthcare system and pharmaceutical industry are facing major challenges due to the economic and social changes (5). In this context, in 2013, two analyses developed under the aegis of the Center for Disease and Control and Prevention (CDC) highlighted the severe human and economic burden associated with sexually transmitted infections (STIs) in the United States $(6,7)$. These two studies estimated the number of new STI cases per year in the United States to be approximately 20 million, and estimated the total lifetime direct medical costs for these new cases of STIs to reach to almost 16 billion USD. While most of these STIs will not cause harm, some have the potential to cause serious health problems, particularly if not diagnosed and treated at an early stage (8). In our study, in our female population, the high-risk HPV strains were found in the patients who were $<35$ years of age. While the vast majority of HPV infections will self heal within 2 years and cause no harm, some of these infections will take hold and potentially lead to serious disease, including cervical cancer $(8,9)$. Although there is also no generally accepted definition of HPV persistence (10), the most commonly used definition is two or more HPV DNA-positive tests during the follow-up $(11,12)$.

HPV16 and 18 are the predominant oncogenic genotypes, involved in the development of $70 \%$ of global cervical cancer cases $(13,14)$, with the exception of women infected with HIV, in whom HPV58 is reported to be the second most dominant strain behind HPV16 (15). In our study, no HIV subjects were enrolled and the predominant strain in female subjects was strain 16 , followed by strains 52,51 and 18 . It has been reported that the majority of young females with oral HPV infection have cervical HPV infection with type concordance and dominance of HPV16 $(16,17)$. In this aspect, the continuation of our study will be the identification of high-risk strains in both cervical and oral sites. As the literature is citing extremely diverse associations, different results in studies may be due to the different populations, biological sampling methods and the assays used $(16,18)$.

However, for neoplastic transformation to take place, apart from HPV infection, a number of putative viral, host and environmental co-factors, such as ionizing irradiation, UV exposure, as well as mechanical and chemical stresses are required (19). As we found in our female population, there was a significant proportion of subjects exhibiting a combination of high- and low-risk HPV strains; thus, we cannot rule out the possibility of the association of low-risk strains with the risk of neoplastic transformation.

HPV is a leading cause of anogenital malignancies and a role of HPV in the etiology of oro-pharyngeal cancers has been demonstrated. The frequency of oral HPV infection in patients with genital warts and the association between concomitant genital, anal and oral infection is unclear. Kofoed et al recruited a total of 201 men and women with genital wart-like lesions. Swab samples were obtained from the genital warts and the anal canal and an oral rinse was collected. Anal HPV was found in $46.2 \%$ and oral HPV in $10.4 \%$ of the participants. The concordance between anal and genital wart HPV types was $78.1 \%$, while the concordance between oral and genital wart types was $60.9 \%$. A lower concordance of $21.7 \%$ was observed between the anal and oral HPV types. Significantly more women than men had multiple HPV types and anal HPV (20). In our study, all enrolled patients were diagnosed with genital warts.

In conclusion, in this study $>700$ female subjects were tested for high- and low-risk genital HPV. We found that the high-risk strains were mainly identified in the patients $<35$ years of age. The incidence of HPV in the human cancer domain is still a subject of intensive investigation. There are areas in the field of HPV infection in which there are still ongoing debates and conclusions have not yet been reached. For example, there is still debate as to the involvement of stresses in triggering dermatological conditions (21) and/or the involvement of stresses in triggering an $\mathrm{HPV}^{+}$lesion to evolve into neoplasia (22-24). Chemically-induced pro-tumoral factors, drugs and environmental milieu (25), represent another domain that could explain $\mathrm{HPV}^{+}$-triggering conditions. Last but not least, the omics domain (26) may bring additional data into this field, as there are data showing a pro-tumoral association of $\mathrm{HPV}^{+}$lesions with miRNA profiles and/or particular proteomics patterns (27).

\section{Acknowledgements}

The authors would like to thank the Medicover Healthcare Centre (Bucharest, Romania) for granting us access to their database.

\section{References}

1. Škamperle M, Kocjan BJ, Maver PJ, Seme K and Poljak M: Human papillomavirus (HPV) prevalence and HPV type distribution in cervical, vulvar, and anal cancers in central and eastern Europe. Acta Dermatovenerol Alp Pannonica Adriat 22: 1-5, 2013.

2. Voidăzan S, Tarcea M, Morariu SH, Grigore A and Dobreanu M: Human papillomavirus vaccine - knowledge and attitudes among parents of children aged 10-14 years: A cross-sectional study, Tirgu Mures, Romania. Cent Eur J Public Health 24: 29-38, 2016. 
3. Salavastru CM, Niculescu MC, Zota A, Nicola G, Morariu HS, Solovan C, Patrascu V, Popovici G, Vladuta R, Panduru M, et al: Epidemiological aspects of genital warts in romania - a 2012 retrospective survey. Maedica (Buchar) 9: 144-150, 2014.

4. Gravitt PE, Peyton CL, Alessi TQ, Wheeler CM, Coutlée F, Hildesheim A, Schiffman MH, Scott DR and Apple RJ: Improved amplification of genital human papillomaviruses. J Clin Microbiol 38: 357-361, 2000.

5. Raţiu MP, Purcărea I, Popa F, Purcărea VL, Purcărea TV, Lupuleasa D and Boda D: Escaping the economic turn down through performing employees, creative leaders and growth driver capabilities in the Romanian pharmaceutical industry. Farmacia 59: 119-130, 2011

6. Satterwhite CL, Torrone E, Meites E, Dunne EF, Mahajan R, Ocfemia MC, Su J, Xu F and Weinstock H: Sexually transmitted infections among US women and men: Prevalence and incidence estimates, 2008. Sex Transm Dis 40: 187-193, 2013.

7. Owusu-Edusei K Jr, Chesson HW, Gift TL, Tao G, Mahajan R, Ocfemia MC and Kent CK. The estimated direct medical cost of selected sexually transmitted infections in the United States, 2008. Sex Transm Dis Mar 40: 197-201, 2013.

8. Workowski KA and Berman S; Centers for Disease Control and Prevention (CDC): Sexually transmitted diseases treatment guidelines, 2010. MMWR Recomm Rep 59 (RR-12): 1-110, 2010

9. Koshiol J, Lindsay L, Pimenta JM, Poole C, Jenkins D and Smith JS: Persistent human papillomavirus infection and cervical neoplasia: A systematic review and meta-analysis. Am J Epidemiol 168: 123-137, 2008.

10. Louvanto K, Rintala MA, Syrjänen KJ, Grénman SE and Syrjänen SM: Genotype-specific persistence of genital human papillomavirus (HPV) infections in women followed for 6 years in the Finnish Family HPV Study. J Infect Dis 202: 436-444, 2010.

11. Kjær SK, Frederiksen K, Munk C and Iftner T: Long-term absolute risk of cervical intraepithelial neoplasia grade 3 or worse following human papillomavirus infection: Role of persistence. J Natl Cancer Inst 102: 1478-1488, 2010.

12. Syrjänen K, Shabalova I, Naud P, Kozachenko V, Derchain S, Zakharchenko S, Roteli-Martins C,Nerovjna R,Longatto-Filho A, Kljukina L, et al; NIS and LAMS Study Research Groups: Risk estimates for persistent high-risk human papillomavirus infections as surrogate endpoints of progressive cervical disease critically depend on reference category: Analysis of the combined prospective cohort of the New Independent States of the Former Soviet Union and Latin American Screening studies. Int J STD AIDS 22: 315-323, 2011.

13. Li N, Franceschi S, Howell-Jones R, Snijders PJ and Clifford GM: Human papillomavirus type distribution in 30,848 invasive cervical cancers worldwide: Variation by geographical region, histological type and year of publication. Int J Cancer 128 927-935, 2011.
14. Mammas IN and Spandidos DA: Fighting against human papillomavirus: the 25-year old contribution of the University of Crete School of Medicine. J BUON 20: 17-21, 2015.

15. Clifford GM, Gonçalves MA and Franceschi S; HPV and HIV Study Group: Human papillomavirus types among women infected with HIV: A meta-analysis. AIDS 20: 2337-2344, 2006.

16. Du J, Nordfors C, Ährlund-Richter A, Sobkowiak M, Romanitan M, Näsman A, Andersson S, Ramqvist T and Dalianis T: Prevalence of oral human papillomavirus infection among youth, Sweden. Emerg Infect Dis 18: 1468-1471, 2012.

17. Giraldo P, Gonçalves AK, Pereira SA, Barros-Mazon S, Gondo ML and Witkin SS: Human papillomavirus in the oral mucosa of women with genital human papillomavirus lesions. Eur J Obstet Gynecol Reprod Biol 126: 104-106, 2006.

18. Termine N, Giovannelli L, Matranga D, Caleca MP, Bellavia C, Perino A and Campisi G: Oral human papillomavirus infection in women with cervical HPV infection: New data from an Italian cohort and a metanalysis of the literature. Oral Oncol 47: 244-250, 2011

19. De Marco F: Oxidative stress and HPV carcinogenesis. Viruses 5: 708-731, 2013.

20. Kofoed K, Sand C, Forslund O and Madsen K: Prevalence of human papillomavirus in anal and oral sites among patients with genital warts. Acta Derm Venereol 94: 207-211, 2014.

21. Căruntu C, Grigore C, Căruntu A, Diaconeasa A and Boda D: The role of stress in skin disease. Intern Med 8: 73-84, 2011.

22. Fang CY, Miller SM, Bovbjerg DH, Bergman C, Edelson MI, Rosenblum NG, Bove BA, Godwin AK, Campbell DE and Douglas SD: Perceived stress is associated with impaired T-cell response to HPV16 in women with cervical dysplasia. Ann Behav Med 35: 87-96, 2008.

23. Spandidos DA: A unified theory for the development of cancer. Biosci Rep 6: 691-708, 1986.

24. Spandidos DA: The cancer story. Cancer Biol Ther 3: 1184-1186, 2004.

25. Neagu M, Caruntu C, Constantin C, Boda D, Zurac S, Spandidos DA and Tsatsakis AM: Chemically induced skin carcinogenesis: Updates in experimental models (Review). Oncol Rep 35: 2516-2528, 2016.

26. Boda D: Cellomics as integrative omics for cancer. Curr Proteomics 10: 237-245, 2013.

27. Wu DW, Chuang CY, Lin WL, Sung WW, Cheng YW and Lee H: Paxillin promotes tumor progression and predicts survival and relapse in oral cavity squamous cell carcinoma by microRNA-218 targeting. Carcinogenesis 35: 1823-1829, 2014. 